\title{
Role of Coupled Flow Dynamics and Real Network Structures on Hortonian Scaling of Peak Flows
}

\author{
Ricardo Mantilla ${ }^{a, *}$ Vijay Gupta ${ }^{a}$ \\ ${ }^{a}$ Department of Civil and Environmental Engineering, Cooperative Institute for Research \\ in Environmental Sciences, University of Colorado. CB216. Boulder, CO. 80309
}

Oscar Mesa ${ }^{b}$

${ }^{\mathrm{b}}$ Universidad Nacional de Colombia sede Medellín. Medellín, Colombia

\begin{abstract}
We investigate how channel flow dynamics on real river networks produces scaling in peak flows. Scaling relations have been defined by log-log linearity between peak flow statistics and drainage areas for idealized mean self-similar networks like Peano and Mandelbrot Viscek. Unlike idealized basins, drainage areas and link lengths in real basins vary statistically. We use Horton-Strahler order as scale parameter instead of drainage area, and use the Hortonian framework to analyze these network structures. A river-network oriented GIS is used to extract the drainage network for the Walnut Gulch basin, Arizona in the United States. It provides the link connectivity structure and the geometric variables for numerically solving coupled link-based mass and momentum conservation equations. The equations are solved for spatially uniform and instantaneous injection of runoff under three different scenarios of flow in the channels: 1) Constant Velocity, 2) Constant Friction, and 3) Spatially Variable Friction. We find that Hortonian scaling in peak flows does not hold for the constant friction scenario. The scaling exponents of peak flows for the other two cases are larger than the scaling exponent of the peaks of the width functions. This property of scaling exponents for a real network is opposite to previous findings for idealized mean self-similar networks. An empirical scaling analysis of peak flows on the Walnut Gulch basin is briefly explained to provide preliminary empirical support to our theoretical findings.
\end{abstract}

Key words:

Hortonian Scaling of peak flows, Flow dynamics, Real river networks, HidroSig, Problem of Ungauged Basin, PUB 


\section{Introduction}

The problem of fbod prediction across a very wide range of space and time scales in ungauged and poorly gauged basins remains a grand scientifi c challenge. The current approach to this problem is known as the regional fbod-frequency analysis. A large number of papers on this subject have been published since its fi rst introduction in the mid 1940s (see Gupta et al. (1994) for a brief review). However, regional fbod-frequency analyses are based in multiple regressions, and the resulting statistical parameters that describe fbod-risk factors, remain disconnected with the hydrology of rainfall-runoff processes. A link between the two is a critical need for solving many contemporary problems, such as, the effect of anthropogenic and natural variability on estimating fbod-risk factors. As a consequence of such unknowns, future environmental conditions are not generally represented by historic data sets (Jain and Lall, 2001). In this sense, all basins become ungauged, and predictions from such basins using current regional fbod-frequency analyses is impractical.

A scaling theory of fbods on nested channel networks is being developed to provide a process-based understanding of scaling in spatial fbod statistics (Gupta, 2004). Gupta et al. (1996) published the fi rst paper on the statistical scaling theory of fbods on a highly idealized channel network known as Peano basin. Results in Gupta et al. (1996) have been generalized in many directions for idealized mean self-similar channel networks (Gupta and Waymire, 1998; Menabde et al., 2001; Menabde and Sivapalan, 2001; Veitzer and Gupta, 2001; Troutman and Over, 2001; Morrison and Smith, 2001). However, there is a pressing need to further generalize and test the scaling theory of fbods on real channel networks. Without this key step, it would be practically impossible to apply this new theory to real world situations. A generalization of the scaling theory of fbods from idealized networks to real basins requires a major conceptual, analytical leap as explained in Gupta and Mantilla (2005), and a numerical leap as explained here.

A conceptual diffi culty in generalizing the scaling theory to real basins arises from the presence of statistical spatial variability in the topologic, geometric, and hydraulicgeometric properties in real channel networks (Veitzer and Gupta, 2000, 2001; Veitzer et al., 2003). Unlike idealized mean self-similar basins, drainage area is not a convenient scale parameter to investigate scaling in real basins. Recent developments in the statistical theory of channel networks have shown that the well-known Horton-Strahler order is a natural scale parameter for investigating scaling (Veitzer and Gupta, 2000, 2001). Technical details of formulating Hortonian statistical scal-

\footnotetext{
* Corresponding Author

Email addresses: ricardo@cires.colorado.edu (Ricardo Mantilla), guptav@cires.colorado.edu (Vijay Gupta), ojmesa@unalmed.edu.co (Oscar Mesa).

URL: http: //cires.colorado.edu/ ricardo/ (Ricardo Mantilla).
} 
ing of peak fbws on real channel networks is explained in Gupta and Mantilla (2005).

The goal of this paper is to use a new GIS simulation environment called "HidroSig" (Mantilla, 2003) to understand how random spatial variability in real channel network topology, geometry, and dynamics of fbw are coupled to produce scaling in fbods. We describe the simulation environment in section 4 . We use this numerical environment to solve the link-based mass and momentum equations, and then record fbw hydrographs at the end of all complete Horton-Strahler streams on the Walnut Gulch basin, Arizona. This is an experimental basin of the Agricultural Reseach Service (ARS). This allows to test if Hortonian scaling in the mean peak fbw holds, to estimate Horton ratios, and to estimate scaling exponents as explained by Gupta and Mantilla (2005). We consider three different scenarios of fbw dynamics, 1) constant velocity, 2) constant Chezy friction and 3) spatially variable Chezy friction. The results are presented in section 5.

Another goal is to compare our results with previous fi ndings on idealized networks by Gupta et al. (1996); Menabde et al. (2001); Menabde and Sivapalan (2001) and Troutman and Over (2001). It was found in these studies that the scaling structure of the Width Function maxima played a key role in determining the fbod scaling exponent. We present a scaling analysis of the Width Function Maxima for Walnut Gulch basin in section 3.1. We contrast the results obtained in idealized river networks, such as the Peano and the Mandelbrot Viseck trees, with our results in real networks.

We begin this paper with a brief summary in section 2 of key results that have been obtained for idealized mean self-similar networks. Section 3 analyses the channel network geomorphology of the Walnut Gulch basin. It introduces some terminology, and gives some background on estimation of scaling exponent of the peak of the width function following Veitzer and Gupta (2001). Section 4 describes HidroSig, our GIS numerical simulation environment, and its application to solving link-based mass and momentum balance equations on the Walnut Gulch. Section 5 explains the analysis of peak fbw simulations for constant velocity, constant Chezy friction and a spatially variable Chezy friction, and computes fbod scaling exponents using Hortonian analysis. Section 6 gives a qualitative comparison of our numerical results on the fbod scaling exponent with empirical exponents from scaling of annual peak fbws (Goodrich et al., 1997). The paper is concluded with a summary of main fi ndings and challenges for the future in this new area of research.

\section{A Brief Review of the Peak Flow Scaling Results for Idealized Networks}

Scaling concepts require specifi cation of a scale, which depends on the context. For example, the drainage area, $A$ has been used as the scale parameter in three types of 
empirical scaling analyses of fbod peaks. A random process indexed by $A$ exhibits statistical simple scaling if, (i) log-log linearity between quantiles and drainage areas holds, and (ii) the slope remains the same with respect to different recurrence intervals. Another concept, called multi-scaling satisfi es (i), but can accommodate a change in slope with respect to the recurrence interval. The mathematical concepts of scaling have been explained in a self-contained manner by Gupta and Waymire (1998).

Gupta et al. (1996) analyzed scaling in peak fbws on the Peano network under the assumptions that rainfall is injected instantaneously on the network without any infi ltration, and the water moved with a spatially uniform velocity without any fbw attenuation. For the concepts of mean self-similar and deterministic recursive replacement networks, the reader may refer to Peckham (1995) and citetveit2000. Gupta et al. (1996) showed that scaling in peak fbw holds with a scaling exponent $\phi_{2}=\beta=\log 3 / \log 4$, where, $\beta$ is the scaling exponent for the peaks of the width functions in a Peano network. Troutman and Over (2001) generalized this result to the broad class of deterministic recursive replacement networks of which Peano is a special case. Using numerical simulations, Menabde and Sivapalan (2001) extended the above result of Gupta et al. (1996) by incorporating fbw attenuation due to a change of storage in fbw dynamics on a network. They analyzed three channel networks, namely the Peano network, the Mandelbrot-Viscek network and a new class of models called random self similar networks (RSNs) introduced by Veitzer and Gupta (2000). They found that the peak fbws exhibit asymptotic scaling as area becomes large. Moreover, the fbod-scaling exponent was found to be below the value of the scaling exponent for the peaks of the width functions, i.e., $\phi_{2}<\beta$. Menabde and Sivapalan (2001) further generalized this result by assuming that fbw velocity is spatially variable and obeys a nonlinear Chezy relationship in each channel link, $v=C R^{1 / 2} \gamma^{1 / 2}$, where $R$ is the hydraulic radius and $\gamma$ is the channel slope. They found for the Mandelbrot-Viscek network that the scaling exponent $\phi_{2}$ is further reduced below its value for a linear velocity, which was already below $\beta$ as mentioned above. These results are signifi cant because they showed that scaling holds asymptotically under much more realistic fbw dynamics than considered by Gupta et al. (1996). Moreover, these results showed how an interaction between network geometry and fbw dynamics yields the fbod-scaling exponent $\phi_{2}$. A recent review of progress in the scaling theory of fbods is given by Gupta (2004).

In context of the above results, we address two questions, (i) Does scaling in peak fbws hold in real networks for realistic fbw dynamics if rainfall is injected instantaneously on the network without any infi ltration and processes of runoff generation, (ii) does the result $\phi_{2}<\beta$ hold for real networks? This paper furnishes a fi rst major step in meeting numerous challenges that arise in generalizing the scaling results from idealized to real networks. 


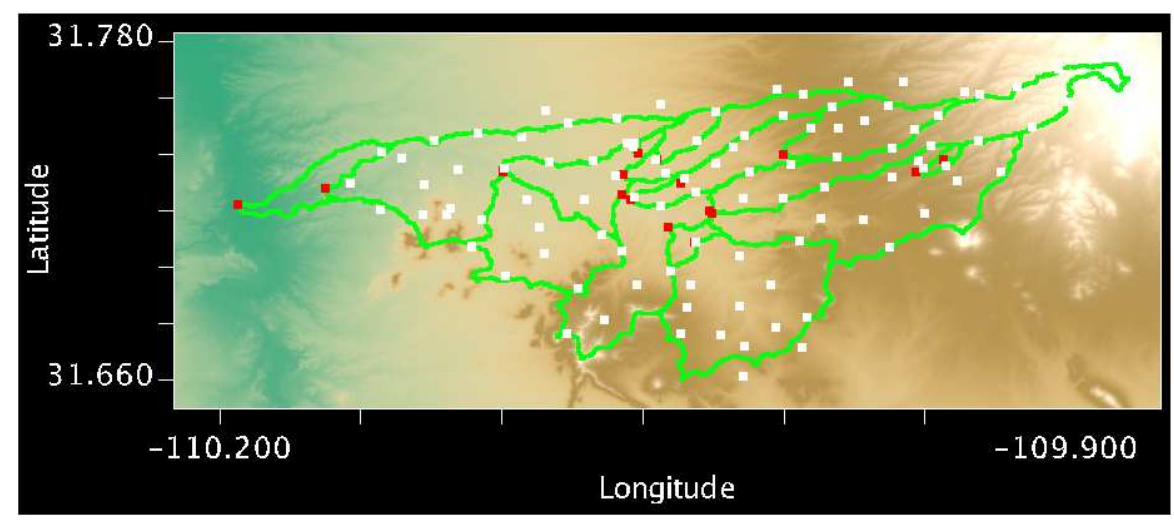

Fig. 1. Location of precipitation gauges (white dots) and streamflow gauges (red dots) inside Walnut Gulch Basin, AZ. The green lines mark the subbasin of each of the stream flow gauges. The basin outlet is at the first red dot on the left hand side of the graph.

\section{Geomorphologic Analyses for the Walnut Gulch basin, Arizona}

\subsection{The Walnut Gulch Network Structure}

Walnut Gulch basin is a $150 \mathrm{~km}^{2}$ experimental watershed located in Arizona. This basin has been used in numerous hydrological studies (Renard et al., 1993; Goodrich et al., 1997). The basin as shown in fi gure 1 is gauged at 18 different locations in the river network (red dots in fi gure 1). The basin is also covered by a dense array of 85 precipitation gauges (white dots in fi gure 1). Walnut Gulch dataset consists of 40 years of stream fbw and rainfall data, from 1957 to 1997. It provides a unique data set for testing peak fbw scaling. For a detailed description of the basin, see Renard et al. (1993).

In order to characterize the basin geomorphology we used HidroSig, a river network oriented GIS developed over the last 5 years by the authors. HidroSig was used to delineate the river network pattern for the Walnut Gulch basin from a $30 \mathrm{~m}$ digital elevation model (DEM) and for decomposing the basin into channel links and hillslopes. Links are defi ned as the section of a river channel between two junctions, a source and a junction or a junction and the outlet. The typical lengths of links vary from about 200 to $500 \mathrm{~m}$. Hillslopes consist of two contributing areas adjacent to each link. Their typical size varies from 0.01 to $0.1 \mathrm{~km}^{2}$. Figure 3 shows the hillslope-link decomposition using HidroSig for the Walnut Gulch basin.

\subsection{Geomorphologic Analysis}

Real channel networks exhibit statistical variability in their topology, geometry (Veitzer and Gupta, 2000) and hydraulic geometry (Ibbitt, 1997; McKerchar et al., 
1998). There is a long history of published literature in geomorphology that has been devoted to understanding this variability (Jarvis and Woldenberg, 1984). Due to random variability in magnitude (or drainage area), it is not a convenient scale parameter for investigating scaling on hierarchical river networks. Rather, the wellknown Horton-Strahler order, $\omega=1,2,3, \ldots$ serves as a natural scale parameter for this purpose. Peckham and Gupta (1999) reformulated the well-known Hortonian relationships in terms of Statistical Self Similarity (SSS) by generalizing Hortonian relationships from means to full probability distributions. Veitzer and Gupta (2000) showed analytically that a broad subclass of RSNs exhibit SSS in magnitudes, areas and Strahler steam lengths. They also showed analytically that under certain conditions RSNs exhibit Tokunaga self-similarity that has been observed for real networks Peckham (1995). We will not discuss the idea of SSS in this paper, but we will use some of the results from Veitzer and Gupta (2001); Gupta and Mantilla (2005) to compute scaling exponents.

Let $X_{\omega}$ denote a generic random fi eld indexed by Horton-Strahler order, $\omega=$ $1,2,3, \ldots$, which represents any one of the three variables mentioned above. SSS requires that the means, $E\left[X_{\omega}\right], \omega \geq 1$, obey a Hortonian relationship given by,

$$
\frac{E\left[X_{\omega+1}\right]}{E\left[X_{\omega}\right]}=R_{X}, \quad \omega \geq 1
$$

where $R_{X}$ is the Horton ratio for $X_{\omega}$. Cumulative drainage areas, $A_{\omega}$ in the Walnut Gulch basin obey a Hortonian relationship in the means with the Horton ratio, $R_{A}=4.71$ as shown in Fig. 2. SSS is discussed in detail by Veitzer and Gupta (2000), and Veitzer et al. (2003) both empirically and for RSNs. Veitzer and Gupta (2001) investigated SSS in the peaks of the width functions, $\Theta_{\omega}, \omega=1,2,3, \ldots$ in detail. They suggested that a scaling exponent, $\beta$ for the peaks of the width functions can be computed from the relationship,

$$
\beta=\log R_{\Theta} / \log R_{A}
$$

Figure 2.b. shows the Hortonian relationship for the means, $E\left[\Theta_{\omega}\right], \omega \geq 1$, which gives a Horton ratio, $R_{\Theta}=2.12$. Therefore, eq. 2 predicts, $\beta=0.485$ for the Walnut Gulch basin.

\section{Description of the Numerical Simulation Laboratory}

An analytic solution of the coupled mass and momentum conservation equations on real channel networks to predict statistical scaling in peak fbws is a diffi cult mathematical problem (Troutman and Over, 2001). For example, Menabde et al. (2001) could not obtain an analytical solution of the mass conservation equation 


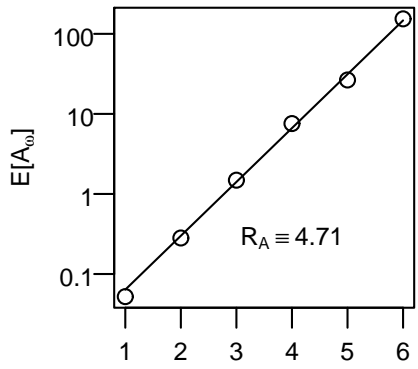

$\omega$

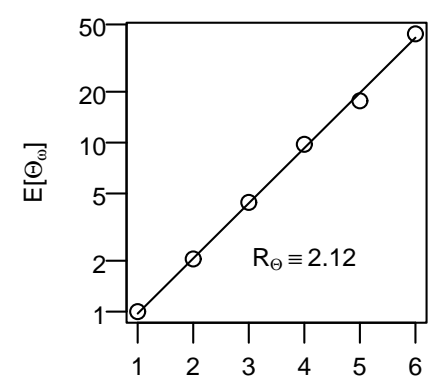

$\omega$

Fig. 2. Hortonian scaling test for (a) means of drainage areas and (b) means of peaks of Topologic Width Functions.

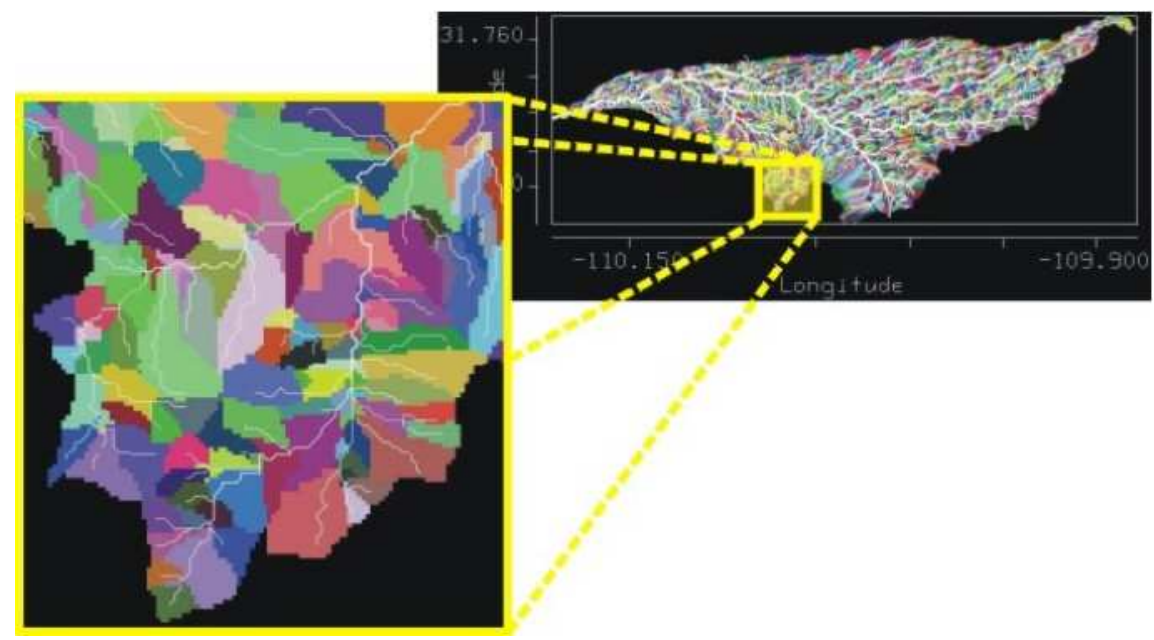

Fig. 3. The Walnut Gulch basin decomposed in hillslopes and links by HidroSig.

for constant velocity in presence of fbw attenuation due to a change of storage on a Peano network. Even for this highly idealized case, a numerical approach had to be used. We adopt a numerical approach to solve the link-based mass conservation equation proposed by Gupta and Waymire (1998). This formulation was extended to include momentum, energy, and entropy conservation by Reggiani et al. (2001). Our numerical model uses connectivity among links of a channel network that is extracted using HidroSig described in the previous section. The data processing for the channel network provides the network connectivity structure, and it also decomposes a basin into hillslopes. A hillslope-link based decomposition of a river basin provides the simulation environment for runoff generation from hillslopes to links, and for transport through the network. 


\subsection{Flow in Channels}

In the presence of statistical spatial variability in link lengths and space-time variable fbw velocity in real networks, it is inconvenient to treat time as discrete because a characteristic time scale for the entire network is not apparent. Therefore, we replace discrete time by a continuous time in the original mass conservation equation (Gupta and Waymire, 1998). Notice that $\Delta t \rightarrow d t$ implies that depth is the same across a single link at any instant of time, $t$. A relationship between fbw velocity and depth is determined by the momentum equation, which is necessary for solving the mass balance equation. We simplify our momentum equation by assuming that frictional and gravitational forces are in equilibrium at all time and follow a Chezy equation $v=C R^{1 / 2} \gamma^{1 / 2}$. We replace the hydraulic radius, $R$ with the channel depth, $d$. This equation is consistent with the velocity profi le for a free surface turbulent fbw (Reggiani et al., 2001).

For continuous time, the mass conservation equation for a link, $e$ can be written as,

$$
\frac{d S(e, t)}{d t}=a_{e} R(e, t)+q\left(f_{1}, t\right)+q\left(f_{2}, t\right)-q(e, t)
$$

where, $S(e, t)$ is the storage in the link at time $t, a_{e}$ is the total hillslope area draining into the link, $R(e, t)$ is the runoff intensity per unit area from the hillslope, and $q\left(f_{1}, t\right)+q\left(f_{2}, t\right)$ are the fbws from the two upstream tributaries joining the link $e$. We also assume for simplicity that link has a rectangular x-section, so the width, $w_{e}$, does not change with time. Therefore, we can write the channel storage, $S(e, t)$, and discharge, $q(e, t)$, as $S(e, t)=l_{e} w_{e} d_{e}(t)$ and $q(e, t)=v_{e}(t) w_{e} d_{e}(t)$, where $w_{e}$ is the mean width of the link, $d_{e}(t)$ the mean channel depth, $v_{e}(t)$ is the $\mathrm{fbw}$ velocity, and $l_{e}$ is the link length. Combining these two equations gives

$$
S(e, t)=\frac{q(e, t) l_{e}}{v_{e}(t)}
$$

Finally, substituting Chezy equation into 4, and approximating $R_{e}$ by $d_{e}$, we get channel storage as a function of discharge, $S(e, t)=q(e, t)^{\frac{2}{3}} C_{e}^{-\frac{2}{3}} w_{e}^{\frac{1}{3}} l_{e} \gamma_{e}^{-\frac{1}{3}}$.

Since, $C_{e}, w_{e}, l_{e}$, and $\gamma_{e}$ are constant in time, we can differentiate the above equation with respect to time to get

$$
\frac{d S(e, t)}{d t}=\frac{2}{3} q(e, t)^{-\frac{1}{3}} \frac{d q(e, t)}{d t} C_{e}^{-\frac{2}{3}} w_{e}^{\frac{1}{3}} l_{e} \gamma_{e}^{-\frac{1}{3}}
$$

substituting 5 into 3 gives a nonlinear differential equation governing fbw dynam- 
ics in a channel network as,

$$
\frac{d q(e, t)}{d t}=K(q(e, t))\left[a_{e} R(e, t)+q\left(f_{1}, t\right)+q\left(f_{2}, t\right)-q(e, t)\right]
$$

where $K(q(e, t))=\frac{3}{2} q(e, t)^{1 / 3} C_{e}^{2 / 3} w_{e}^{-1 / 3} l_{e}^{-1} \gamma_{e}^{1 / 3}$. Notice that this equation can be further simplifi ed if, instead of Chezy equation, we assume a constant velocity for all times. Equation 4 reduces to $\frac{d S_{l}}{d t}=\frac{l_{e}}{v} \frac{d q(e, t)}{d t}$, hence $K(q(e, t))=v / l_{e}$. The above equation can be generalized to include a more complex formulation of the momentum equation than presented here. For example, one can assume more realistic channel x-sections than rectangular, and include fbw over fbod plains (Kean, 2003).

The term $R(e, t)$ represents fbw from hillslopes into the link $e$ in the set of equations 6 describing the channel fbw dynamics. In order to complete the formulation to simulate rainfall-runoff process in the basin, equations describing runoff generation from hillslopes is required, see, e.g.,(Duffy, 1996)). Since our objective is to understand the role of network geometry and dynamics on the fbod scaling exponent, $\phi_{2}$, we only need to consider instantaneous injection of runoff on the channel network as explained in the section 2. Consequently, we will assume that the runoff rate is known and has a very short duration $(\sim 1 \mathrm{~min})$ representing instantaneous injection. In the next section we explain our method for determining runoff rates on the Walnut Gulch.

\subsection{Runoff Generation on Walnut Gulch}

Walnut Gulch is a semi-arid basin in which infi ltration dominates in converting rainfall to runoff. We have chosen a simple approach to estimate typical runoff rates in this basin. Our approach assumes that the infi ltration rate, $I_{f}$ is constant during a precipitation event. This assumption is good as a fi rst approximation for semi-arid climates because intense rainfall is mostly produced by convective events, which generally do not last for more than 20-40 minutes.

In order to estimate runoff rates, we performed a water balance analysis on the two smallest gauged subbasins of Walnut Gulch with drainage areas of $0.055 \mathrm{~km}^{2}$ and $0.064 \mathrm{~km}^{2}$. The entire analysis assumes that precipitation doesn't vary in space within these subbasins. Therefore, a single rainfall gauge is used to estimate total volume of rainfall on each of these two basins for the water balance analysis.

The water balance equation for the basin can be written as

$$
R=P-I
$$



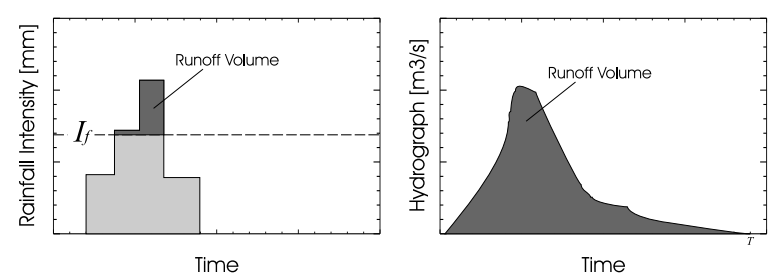

Fig. 4. A schematic depiction of how the infiltration intensity $I_{f}$ is estimated, to obtain a time series of runoff intensities.
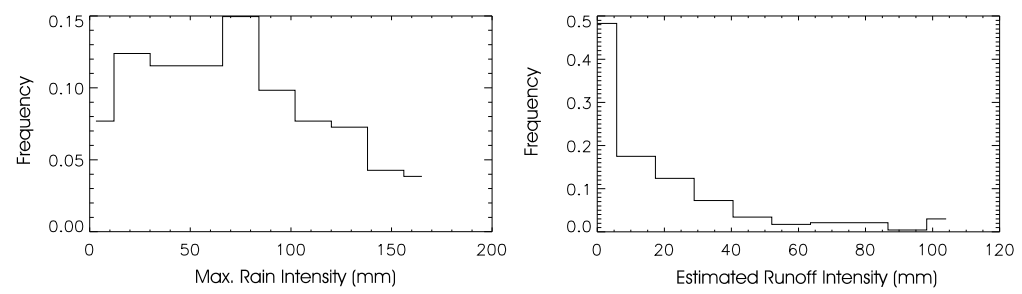

Fig. 5. Histogram of maximum intensities and estimated runoff rates for 250 rainfall-runoff events in two small sub basins in Walnut Gulch basin.

where $R$ is total observed runoff, $P$ is total rainfall, and $I$ is total infi ltrated volume of water from a single rainfall event of duration $T$. Since the infi ltration rate is assumed to be a constant, total runoff can be defi ned as

$$
R=\int_{0}^{T} r_{t} d t \quad \text {, and } \quad r_{t}=\left\{\begin{array}{lll}
i_{t}-I_{f} & \text {,if } & i_{t}>I_{f} \\
0 & \text {,if } & i_{t}<I_{f}
\end{array}\right.
$$

Notice from equation 8 , that given runoff volume, $R$, and a rainfall intensity time series, $i_{t}$, it is possible to fi nd the value, $I_{f}$ that satisfi es equation 7 . This equation needs to be solved numerically due to complexity in the rainfall intensity time series, $i_{t}$. Figure 4 illustrates this concept, which is well known in literature as the $\phi$-index method of infi ltration (Chow et al., 1988).

We estimated runoff from a total of 250 events in the two subbasins mentioned above. The histograms for maximum precipitation rate and estimated runoff rates are given in fi gure 5. The estimated expected value of runoff intensity is $12 \mathrm{~mm} / \mathrm{h}$ and the standard deviation is $15 \mathrm{~mm} / \mathrm{h}$. These runoff rates are typical for Walnut Gulch from hillslopes that feed into links. We use these values in our numerical simulations described below.

\subsection{Numerical Solutions of Coupled set of dynamical equations on a Channel network}

The link-based mass conservation equation leads to a system of $M$ nonlinear coupled ordinary differential equations (ODEs) where $M$ is the number of links in the 


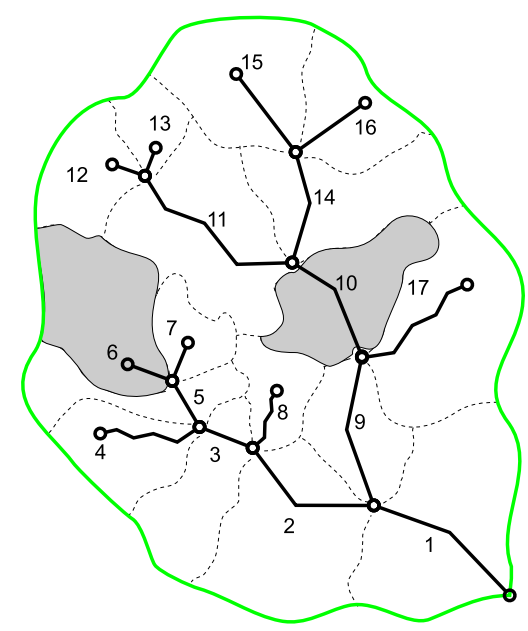

Fig. 6. A generic river basin decomposed in links and hillslopes. Each link is labeled.

network. As an example, consider the basin in fi gure 6. For the external link number 6 , the mass conservation equation can be written as:

$$
\frac{d q(6, t)}{d t}=K(q(6, t))\left[a_{6} P(6, t)-q(6, t)\right]
$$

where $K(q(6, t))=\frac{3}{2} q(6, t)^{1 / 3} C_{6}^{2 / 3} w_{6}^{-1 / 3} l_{6}^{-1} \gamma_{6}^{1 / 3}$

For link number 10, an internal link with link numbers 11 and 14 draining into it at its upper junction, the mass conservation equation is given by:

$$
\begin{aligned}
\frac{d q(10, t)}{d t}= & K(q(10, t))\left[a_{10} R(10, t)\right. \\
& +q(11, t)+q(14, t)-q(10, t)]
\end{aligned}
$$

where $K(q(10, t))=\frac{3}{2} q(10, t)^{1 / 3} C_{10}^{2 / 3} w_{10}^{-1 / 3} l_{10}^{-1} \gamma_{10}^{1 / 3}$

In summary, a set of non-linear coupled ODEs of the form

$$
\frac{d \underline{q}}{d t}=f(\underline{q}, t)
$$

describes runoff dynamics on the channel network that produces fbods. Such a system can be solved numerically using a truncated Taylor series expansion for $f(\underline{q}, t)$. We have chosen the Runge-Kutta-Fehlberg algorithm that provides a fi fth order approximation to the solution of this problem. It was implemented with an adaptive time step control algorithm based on the worst offender proposed by Cash and Karp (1990). 


\subsection{Parameterization of the Runoff DynamicsModel}

The system of ODEs describing runoff dynamics contain four parameters per link, namely, channel slope, $\gamma$, link length, $l$, Chezy coeffi cient, $C$, and channel width, $w$. The fi rst two parameters can be measured directly from a DEM but the last two need proper parameterization. This issue requires ideas of spatial variability of Hydraulic Geometric ( $\mathrm{H}-\mathrm{G})$ variables given by width $w$, depth $d$, velocity $v$, slope $\gamma$, friction coeffi cient $C$ and sediment transport $Q_{s}$ as power law functions of some basin property such as mean discharge or drainage area. Some of the most recent observations on downstream $\mathrm{H}-\mathrm{G}$ on Walnut Gulch were analyzed by Miller (1995). He found the relation $w=2.36 A_{c}^{0.34}$ for the channel width $w$ with respect to the drainage area $A_{c}$.

In the numerical simulations given in the next section, we used two different assumptions on Chezy coeffi cient, $C$. First, we assume that it is constant, and second that it can be parameterized as a function of channel slope, $\gamma$ for each link. We use some empirical relationships by Strickler (1923) and Shields (1936) to derive a relationship between $C$ and $\gamma$. We used these two because no spatial observations about $C$ are available for the Walnut Gulch.

\section{Tests of Scaling in Peak Flows using Dynamical Simulations}

Our fi rst goal here is to illustrate using numerical simulations how Hortonian scaling in peak fbws arises from dynamics of runoff on the Walnut Gulch basin, as

$$
\frac{E\left[Q_{\omega}\right]}{E\left[Q_{\omega-1}\right]}=R_{Q}, \quad \omega=1,2,3, \ldots
$$

The second goal is to compute the fbod scaling exponent $\phi_{2}$ given by Gupta and Mantilla (2005), where if a Horton type law given by

$$
\phi_{2}=\frac{\log \left(R_{Q}\right)}{\log \left(R_{A}\right)}
$$

Using the numerical model described in section 4 we simulate discharge hydrographs for every link in the basin for an instantaneous $(\sim 1 \mathrm{~min})$ injection of runoff rate on the network, and compute peak fbws. Our main task is to test if a Hortonian relationship holds for mean peak fbws obtained from simulated hydrographs under three different assumptions about the velocity. Our three assumptions are the same that have been previously used on idealized networks, namely 1) Constant Velocity, 2) Constant Chezy, and 3) Spatially variable Chezy obeying a H-G relationship (Menabde et al., 2001; Menabde and Sivapalan, 2001). 

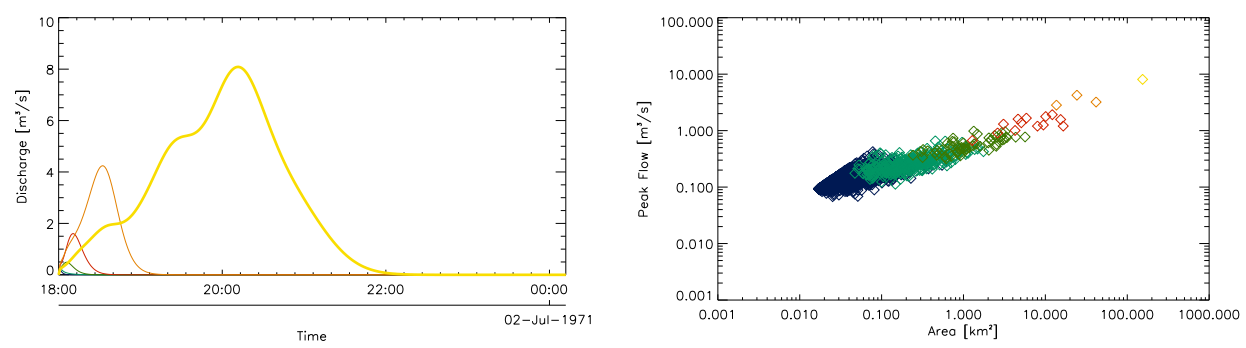

Fig. 7. (a) Hydrographs for different order streams, and (b) Peak Flows vs. Area, from simulations using the constant velocity assumption.

\subsection{Constant Velocity Scenario}

Under the assumption that velocity is constant in time and space, equation 6 can be further simplifi ed as $K(q(e, t))=v / l$, where $v$ is the velocity of fbw ( $v=2 \mathrm{~m} / \mathrm{s}$ for all channels). Figure 7a shows the hydrographs at the end of six different Horton order streams. We have chosen only one hydrograph for each complete Horton order stream. Figure $7 \mathrm{~b}$ shows mean peak fbws vs. mean basin area for six different Horton-Strahler orders that are used to test a power law relationship. The Horton analysis is shown in fi gure 8. This Hortonian relationship is predicted from runoff dynamics on the Walnut Gulch. According to equation 13, the estimated scaling exponent, $\phi_{2}=0.55$, is greater than the scaling exponent for the peak of the width function $(\beta=0.485)$.

The above fi nding is different from the result for Peano and Mandelbrot-Viscek trees, by Menabde et al. (2001), where the scaling exponent for the width function maxima was dynamically predicted to be larger than for peak fbws. Menabde et al. (2001) reasoned that this was a consequence of hydraulic attenuation, and that the exponent $\beta$ comes about due to an absence of attenuation. However, our analysis on the Walnut Gulch reveals that the structure of real networks plays a fundamental role in determining the exponent $\phi_{2}$. It suggests that the fbod scaling exponent is governed by a competition between attenuation and aggregation of fbws in real basins, and the aggregation is the dominating factor. This scenario was explored further by changing the applied runoff intensity on the network at the initial time. We found that this change has no effect on the value of the exponent, $\phi_{2}$.

\subsection{Constant Chezy Scenario}

If a constant Chezy coeffi cient is assumed for all links, then $K(q(e, t))=$ $\frac{3}{2} q(e, t)^{1 / 3} C_{e}^{2 / 3} w_{e}^{-1 / 3} l_{e}^{-1} \gamma_{e}^{1 / 3}$ as shown in section 4.1 . We assume a value $100 \mathrm{~m}^{1 / 2} / \mathrm{s}$ for the Chezy coeffi cient.

Figure 9a shows the hydrographs at different spatial scales. Figure 10 shows that 


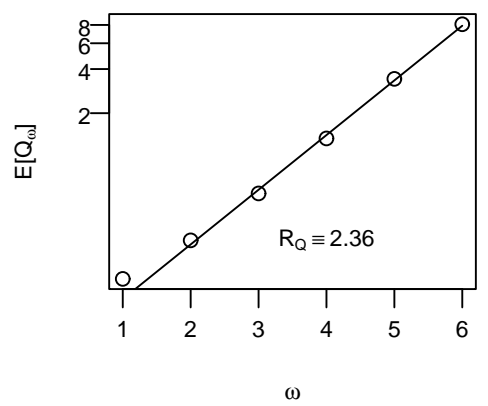

Fig. 8. Horton plot for peak flows using constant velocity.
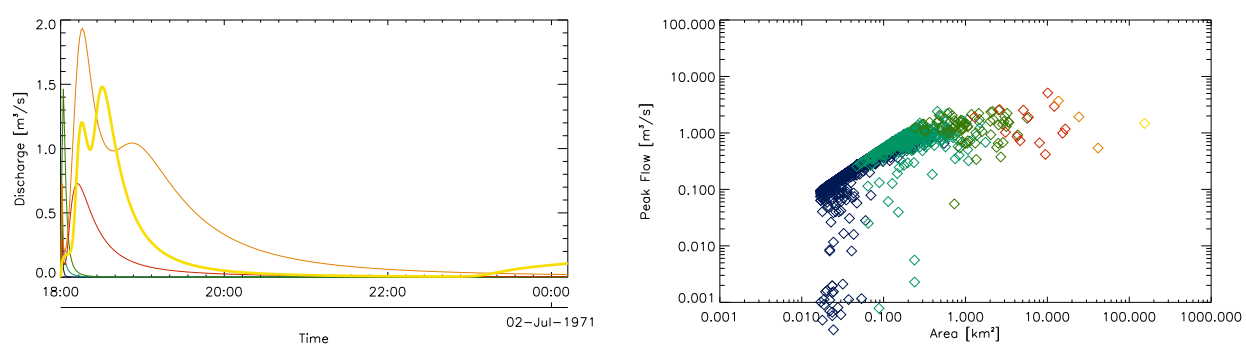

Fig. 9. (a) Hydrographs for different order streams, and (b) Peak Flows vs. Area, from simulations using the constant Chezy assumption.

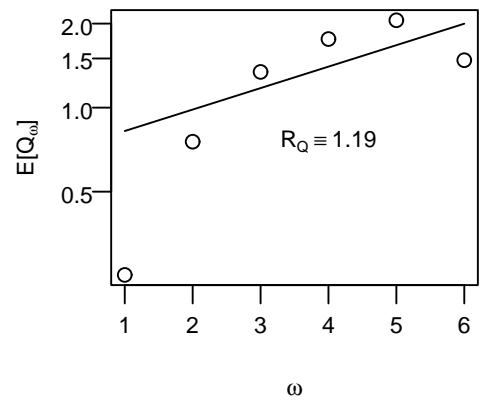

Fig. 10. Horton plot for peak flows using constant Chezy. Since a Horton type law does not hold, the scaling exponent for this scenario cannot be estimated

Horton relationship in peak fbws does not hold. This result is very insightful, because a constant Chezy coeffi cient is an ad hoc simplifi cation that violates Hortonian scaling as observed here. More interestingly, the violation of scaling in peak fbws further demonstrates how it is fundamentally tied to coupling between runoff dynamics and network geometry and topology structure. 


\subsection{Spatially Variable Chezy obeying a H-G Relationship}

We showed at the beginning of this section that the Chezy coeffi cient varies spatially and decreases as a power law function of slope in the downstream direction. We will obtain a similar result from an independent set of empirical relationships from engineering hydraulics so it can be compared with results from downstream $\mathrm{H}-\mathrm{G}$ given earlier. Moreover, we use the result below in our numerical simulations.

Combining results from the initiation of sediment movement with typical empirical values for the critical sheer stress in rivers Shields (1936) found that,

$$
d_{50}=10.2 d \gamma
$$

where $d_{50}, d$ and $\gamma$ are the mean grain size, channel depth and slope, respectively. Strickler (1923) found that Manning's coeffi cient is related to $d_{50}$ as,

$$
n=d_{50}^{1 / 6} / 21
$$

Since Chezy coeffi cient is related to Manning's, $n$, by $C=d^{1 / 6} / n$, we can combine 14 and 15 to write,

$$
C=\frac{d^{1 / 6}}{n}=\frac{21 d^{1 / 6}}{d_{50}^{1 / 6}}=\frac{21 d^{1 / 6}}{10.2^{1 / 6} d^{1 / 6} \gamma^{1 / 6}} \Rightarrow C=14.2 \gamma^{-1 / 6}
$$

We use the above relationship and write $K(q(e, t))$ in equation 6 as

$$
K(q(e, t))=8.8 q(e, t)^{1 / 3} w_{e}^{-1 / 3} l_{e}^{-1} \gamma_{e}^{2 / 9}
$$

The simulated hydrographs for an instantaneous uniform runoff intensity of 20 $\mathrm{mm} / \mathrm{h}$ are presented in fi gure 11a. Hortonian analysis for the peak fbws is shown in fi gure 12. Here, as in the constant velocity scenario, a Hortonian relationship holds. The scaling exponent is $\phi_{2}=0.531$ greater than $\beta$.

To investigate the effect of runoff intensity on the fbod-scaling exponent under this scenario, we repeated our simulations with a $50 \mathrm{~mm} / \mathrm{h}$ runoff intensity. To our surprise, the value of the exponent $\phi_{2}=0.538$ increased with increasing intensity. Table 1 shows the value of the estimated exponent for three different values of runoff intensities. 

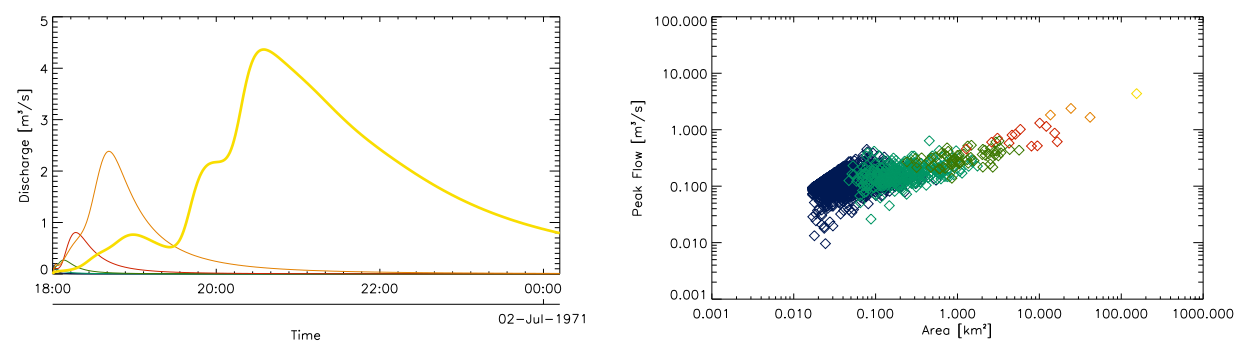

Fig. 11. (a) Hydrographs for different order streams, and (b) Peak Flows vs. Area, from simulations using the variable Chezy assumption.

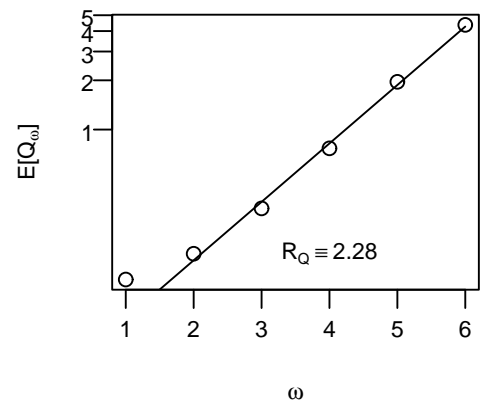

Fig. 12. Horton plot for peak flows using variable Chezy and runoff intensity of $20 \mathrm{~mm} / \mathrm{h}$.

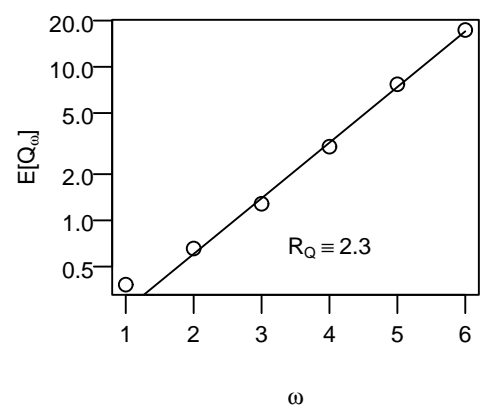

Fig. 13. Horton plot for peak flows using variable Chezy and runoff intensity of $50 \mathrm{~mm} / \mathrm{h}$.

\section{Table 1}

Scaling exponents from simulated peak flows for different runoff intensities

\begin{tabular}{|c|c|}
\hline Rain Intensity $[\mathrm{mm} / \mathrm{h}]$ & Scaling Exponent $\left[\phi_{2}\right]$ \\
\hline \hline 20 & 0.531 \\
\hline 50 & 0.538 \\
\hline 100 & 0.544 \\
\hline
\end{tabular}




\section{A Preliminary Comparison of Empirical Flood Scaling exponent with Nu- merical Results}

Walnut Gulch dataset from 1957 to 1997 contains about 600 rainfall-runoff events ${ }^{1}$. (Goodrich et al., 1997) conducted an empirical scaling analysis of peak fbws on the Walnut Gulch basin. They found log-log linearity between the 2-year and 100year annual fbod quantiles against drainage areas with nearly identical slopes. This suggests the presence of simple scaling in the annual peak fbw data. Gupta (2004) has identifi ed three scaling parameters from this analysis. The fi rst is a critical area, $A_{c}$, around $1 \mathrm{~km}^{2}$ at which the scaling exponent changes from $\theta_{1}$ to $\theta_{2}$. The precise location of scaling break can not be determined from this data set as there is only one stream fbw gage between 0.1 and $2 \mathrm{~km}^{2}$, where the change occurs. Here, $\theta_{1}$ denotes the scaling exponent to the left of break, and $\theta_{2}$ to its right. Both of these parameters describe the scaling statistics of the mean and higher order fi nite moments of peak fbws. The scaling exponent $\theta_{1} \in(0.85,0.90)$ on the left is much larger than $\theta_{2} \in(0.55,0.58)$ on the right.

Our numerical analyses in the previous sections is related to the scaling exponent $\phi_{2}$. However, it is not a straightforward matter to make a direct comparison between the predicted $\phi_{2}$ and $\theta_{2}$. The first issue is that our numerical simulations pertain to a rainfall-runoff event, where as Goodrich et al. (1997) analysis pertains to annual peak fbws. Establishing a precise connection between these two sets of scaling analyses is an open problem for future research. No event-based fbod scaling analyses have been published for the Walnut Gulch basin, and this constitutes an important problem for future research.

The only peak fbw scaling analyses for individual rainfall-runoff events has been conducted on the Goodwin Creek, MS (Ogden and Dawdy, 2003). They observed that plots of peak fbws against drainage areas for individual rainfall-runoff events on the subbasins of the Goodwin Creek show log-log linearity. The regression slope of this relationship can be interpreted as the mean fbod-scaling exponent for an event, say $\phi_{1}$. Among the 223 events that they analyzed, $\phi_{1}$ was found to vary from 0.9 to 0.6 with a mean, $\overline{\phi_{1}}=0.826 \pm 0.047$. Further empirical investigations by Furey and Gupta (2004) on Goodwin Creek have shown that variability in $\phi_{1}$ to a large extent depends on temporal rainfall variability and duration of an event. Ogden and Dawdy (2003) fi gure 2, also found log-log linearity between the 2-year and 20 year annual peak fbws and drainage areas on the Goodwin Creek basin with a slope, $\theta_{1}=0.77 \pm 0.04$. Notice that $\overline{\phi_{1}} \approx \theta_{1}$. It suggests that the mean of event-based scaling exponents and the annual fbod quantile scaling exponent are close to each other but they are not the same.

Based on the above analyses, we conjecture that the mean of the scaling expo-

$\overline{1}$ Dataset available online at http://www.tucson.ars.ag.gov/ 
nents $\phi_{2}$ for individual rainfall-runoff events will be close to the values of $\theta_{2} \in$ $(0.55,0.58)$ obtained by Goodrich et al. (1997). These values are larger than $\beta$, which is consistent with the values obtained from numerical simulations. This result is quite remarkable because it could not have been predicted from previous results on idealized mean self-similar basins. However, we want to stress that beyond this qualitative comparison, it is impossible to make a more specifi c comparison of our results for the present. We are currently investigating event based empirical scaling from data on the Walnut Gulch basin.

\section{Conclusions}

To investigate the coupled role of real river network structures and fbw dynamics on peak fbw scaling exponents, we modifi ed the mass conservation equations proposed by Gupta and Waymire (1998) into a continuous form by letting the travel time in a link, $\Delta t \rightarrow d t$. We solved the resulting set of non-linear ODEs using the Runge-Kutta-Fehlberg algorithm. The network connectivity structure, and geometric information for links and hillslopes was obtained from the output of our own network oriented GIS called HidroSig.

We used the Hortonian framework to estimate scaling exponents for the simulated peak fbws. We found that the resulting scaling exponents reftect different fbw conditions when scaling exists. For example, scaling exists under the assumption of constant velocity in the channels. This result was also found on idealized mean selfsimilar river networks (Menabde et al., 2001). However, peak fbw exponent, $\phi_{2}$ in Walnut Gulch, is larger that the scaling exponent for the peak of the width function, $\beta$. The same result was true when a more realistic fbw dynamics, namely spatially variable Chezy friction was used. This result is opposite to that found by Menabde and Sivapalan (2001) where $\phi_{2}<\beta$. It reveals the fundamental role played by real network structures and fbw dynamics in determining the peak fbw scaling exponents in a basin. We also found that scaling breaks under the assumption of constant Chezy friction, and a scaling exponent cannot be defi ned.

As water fbws downstream in a river network, two effects combine to determine the magnitude of the peak fbw scaling exponent, $\phi_{2}$, relative to the peak of the Width Function scaling exponent, $\beta$. These two effects are attenuation and aggregation. We conclude that in the idealized networks used in previous studies the effect of attenuation was dominant. Notice that the distance from the peak of the width function to the basin outlet is as large as the maximum channel length, which gives a longer travel time to peak fbws over which to attenuate, thus consistently giving $\phi_{2}<\beta$. This is result, as shown here, cannot be expected to hold in real networks, where the peak of the Width Function is usually halfway between the outlet and the longest fbw distance in the basin (Veitzer and Gupta, 2001). 
The results presented here have a direct bearing on the problem of prediction from ungauged basin (PUB), where predictions of peak fbws are required at different spatial and temporal scales. We establish, at least to a fi rst order, that the scaling exponent, $\phi_{2}$, can be tied to network geometry, fbw dynamics and rainfall characteristics. A comprehensive understanding of how these properties interact would be essential in transferring information across different subbasins in a river network.

\section{Acknowledgments}

This research was supported by grants from National Science Foundation. This support is gratefully acknowledged. We also want to thank Peter Furey for his comments during the preparation of this paper.

\section{References}

Cash, J. R., Karp, A. H., 1990. In: ACM Transaction on Mathematical Software. Vol. 16. Association for Computing Machinery, New York, pp. 201-222.

Chow, V. T., Maidment, D. R., Mays, L. W., February 1988. Applied Hydrology. McGraw Hill College Div.

Duffy, C. A., 1996. Two state integral-s balance model for soil moisture and groundwater dynamics in complex terrain. Water Resources Research 32 (8), 2421-2434.

Furey, P., Gupta, V., 2004. Changes in power laws that relate peak discharge to drainage area in a small hortonian basin, preprint, submited to Water Resources Research.

Goodrich, D. C., Lane, L. J., Shillito, R. M., Miller, S., December 1997. Linearity of basin response as a funcition of scale in a semiarid watershed. Water Resources Research 33 (12), 2951-2965.

Gupta, V., Mantilla, R., 2005. Towards a physical theory of hortonian scaling in fbods on real networks, preprint.

Gupta, V., Waymire, E., 1998. Spatial variability and scale invariance in hydrologic regionalization. In: Sposito, G. (Ed.), Scale dependence and scale invariance in hydrology. Cambridge University Press, Cambridge, U.K., pp. 88-135.

Gupta, V. K., January 2004. Emergence of statistical scaling in fbods on channel networks from complex runoff dynamics. Chaos, Solitons \& Fractals 19 (2), 357-365.

Gupta, V. K., Castro, S. L., Over, T. M., 1996. On scaling exponents of spatial peak fbws from rainfall and river network geometry. Journal of Hydrology 187, 81-104.

Gupta, V. K., Mesa, O., Dawdy, D. R., 1994. Multiscaling theory of fbod peaks: regional quantile analysis. Water Resources Research 30 (12), 3405-3421. 
Ibbitt, R. P., 1997. Evaluation of optimal channel network and river basin heterogeneity concepts using measured fbw and channel properties. Journal of Hydrology 196, 119-138.

Jain, S., Lall, U., 2001. Floods in a changing climate: Does the past represent the future? Water Resources Research 27 (12), 3193-3206.

Jarvis, R., Woldenberg, M. (Eds.), 1984. River networks. Vol. 80 of Benchmark papers in geology. Hutchinson Ross, Stroudsburg, Pa.

Kean, J., 2003. Computation of fbw and boundary sheer stress near the banks of streams and rivers. Ph.D. thesis, University of Colorado, Boulder.

Mantilla, R., 2003. A numerical model to explore scaling exponents of fbods. Master's thesis, Universidad Nacional de Colombia sede Medellín, Medellin, Colombia, in Spanish.

McKerchar, A. I., Ibbitt, R. P., Brown, S. L. R., Duncan, M. J., 1998. Data for ahley river to test channel network and river basin heterogeneity concepts. Water Resources Research 34 (1), 139-142.

Menabde, M., Sivapalan, M., 2001. Linking space-time variability of rainfall and runoff fi elds on a river network: A dynamic approach. Advances in Water Resources 24, 1001-1014.

Menabde, M., Veitzer, S., Gupta, V., Sivapalan, M., 2001. Tests of peak fbw scaling in simulated self-similar river networks. Advances in Water Resources 24, 991999.

Miller, S., 1995. An analysis of channel morphology at walnut gulch linking fi eld research with gis applications. Master's thesis, University of Arizona, School of Renewable Natural Resources, available from UMI Dissertation Services.

Morrison, J. A., Smith, J., 2001. Scaling properties of fbod peaks. Extremes 4 (1), 5-22.

Ogden, F. L., Dawdy, D. R., March/April 2003. Peak discharge scaling in small hortonian watershed. Journal of Hydrologic Engineering 8 (2), 64-73.

Peckham, S., 1995. New results for self-similar trees with applications to river networks. Water Resources Research 31 (4), 1023-1029.

Peckham, S., Gupta, V., 1999. A reformulation of Horton's laws for large river networks in terms of statistical self-similarity. Water Resources Research 35 (9), 2763-2777.

Reggiani, P., Sivapalan, M., Hassannizadeh, S. M., Gray, W., 2001. Coupled equations for mass and momentum balance in a stream network: theoretical derivation and computational experiments. Proc. R. Soc. London. A 457, 157-189.

Renard, K. G., Lane, L. J., Simanton, J. R., Emmerich, W. E., Stone, J. J., Weltz, M. A., Goodrich, D. C., Yacowitz, D. S., 1993. Agricultural impacts in an arid environment: Walnut gulch case study. Journal of Hydrology 9 (1-4), 145-190.

Shields, A., 1936. Application of similarity principles and turbulence research to bedload measurement. Tech. Rep. 167, California Institute of Technology, Pasadena.

Strickler, A., 1923. Beiträge zur frage der geschwindigkeits formel und der rauhigkeitszahlen für ströme, kanale und geschlossene leitungen - contributions to the question of a velocity formula and roughness data for streams, channels 
and closed pipelines. Tech. rep., California Institute of Technology, W.M. Keck Laboratory of Hydraulics and Water Resources, translation No. T-10, Translated into English by: Roesgan, T. and Brownie, W.R. (1981).

Troutman, B. M., Over, T. M., 2001. River fbw mass exponents with fractal channel networks and rainfall. Advances in Water ResourcesThis issue.

Veitzer, S., Gupta, V., 2000. Random self-similar river networks and derivations of Horton-type relations exhibiting statistical simple scaling. Water Resources Research 36 (4), 1033-1048.

Veitzer, S., Gupta, V., 2001. Statistical self-similarity of width function maxima with implications to fbods. Advances in Water Resources 24, 955-965.

Veitzer, S., Troutman, B., Gupta, V., 2003. Power-law tail probabilities of drainage areas in river basins. Physical Review E 68. 